九州大学学術情報リポジトリ

Kyushu University Institutional Repository

\title{
The Boron Content of Soils, Plants, And Fertilizer Materials
}

Hirai, Keizo

Biochemical Laboratory, Department of Agriculture, Kyushu University

https://doi.org/10.5109/22613

出版情報：九州大学大学院農学研究院紀要. 9 (1)，pp.83-91，1948-03. Kyushu University バージョン：

権利関係 : 


\title{
THE BORON CONTENT OF SOILS, PLANTS, AND FERTILIZER MATERIALS
}

\author{
Ketzo Hirai
}

\section{INTRODUCTION}

Although there is a very narrow margin between the minute quantities that are necessary and the quantities that are toxic, boron is now recognized as an essential nutrient for normal plant growth.

In plant tissue, boron often is present in fairly large amounts compared with the amounts detectable in soils. With the exception of special cases, boron is not added to the soil as fertilizer, but it is known that various crops absorb it from the soil year after year.

Aware of these facts, and evaluating their importance relative to plant nutrition, it is highly essential that we know the distribution of boron in soils, fertilizers and plants.

In this report, the author presents some facts obtained from his experiments in analysing the distribution of boron in Japanese soils, plants and fertilizer materials.

\section{EXPERIMENTAL METHODS}

To determine the minute quantity of boron, various spectroscopic, titrating (both ordinary and electrometric) and colorimetric methods were introduced by many investigators in the past. After studying some of these methods, the author found that the Berger and Troug method was most suitable for his purpose ; consequently this method has been employed in this study. 
This is the one known as the colorimetric method which employs the reaction between Quinarizarin $(1,2,5,8$, tetrahydroxyl anthraquinon) and boron in concentrated sulfuric acid. (on details, see original.) (1)

\section{RESUlTS}

Deta on the boron content in various Japanese soils are presented in table 1 .

Table 1. Total and Available (Water soluble) Boron Content in various Soils. (p.p.m. in dry basis.)

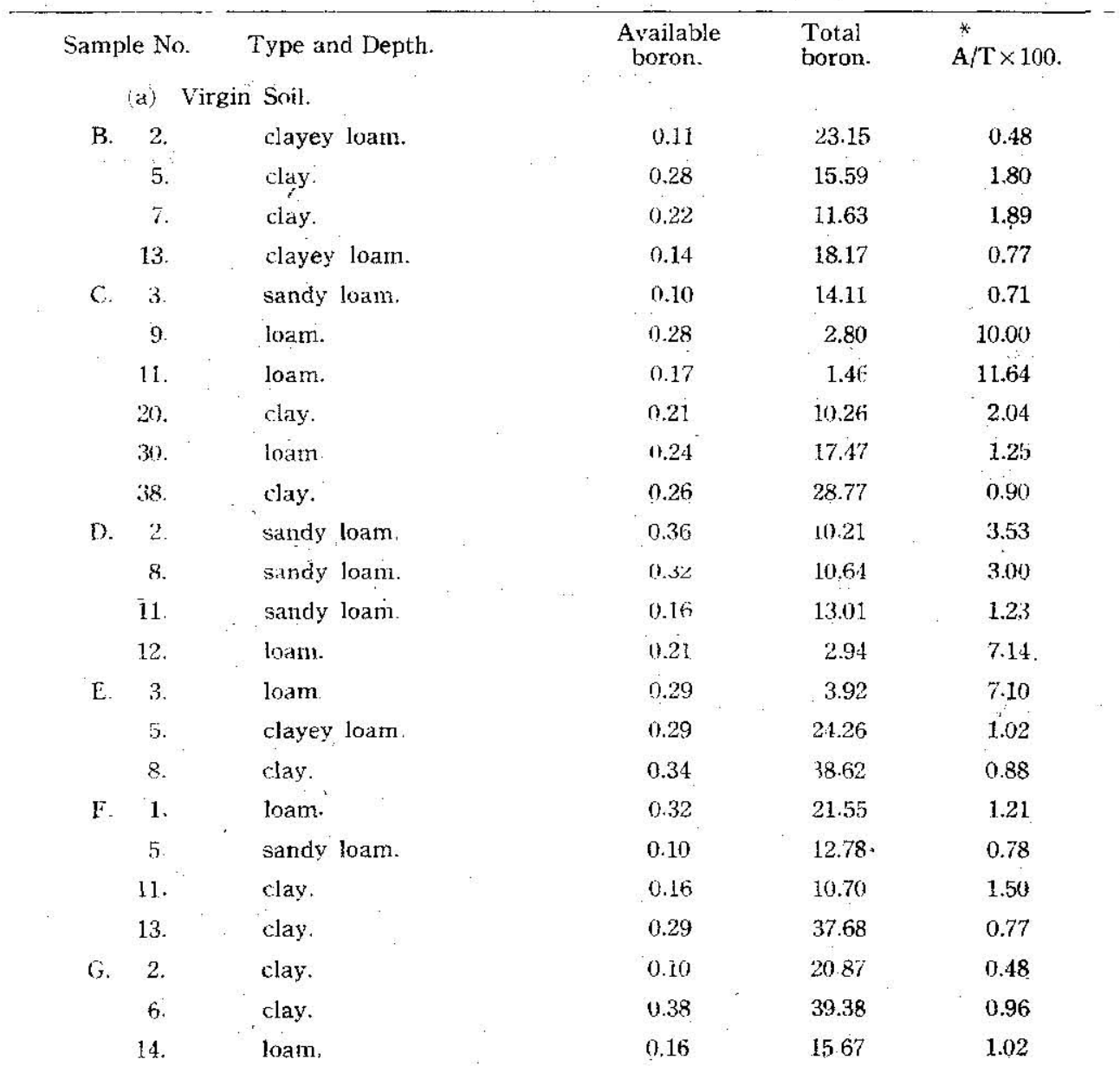




\begin{tabular}{|c|c|c|c|c|c|}
\hline \multirow[t]{4}{*}{$\mathrm{H}$. } & loam. & & 0.11 & 8.15 & 1.34 \\
\hline & loam. & & 0.05 & 11.82 & 0.42 \\
\hline & Ioam. & & 0.21 & 4.02 & 5.22 \\
\hline & clayey loam. & & 0.33 & 16.72 & 1.97 \\
\hline 16. & loam. & & 0.10 & 11.52 & 0.86 \\
\hline \multirow[t]{2}{*}{$\mathrm{K}$. } & clayey loam. & & 0.11 & 2.10 & 5.24 \\
\hline & sandy loam. & & 0.21 & 3.91 & 5.37 \\
\hline 8. & clay. & & 0.32 & 11.45 & 2.72 \\
\hline
\end{tabular}

(b) Arable Soil. (Samples are Fluvial Soil except W 1.-W 5.)

\begin{tabular}{|c|c|c|c|c|c|c|}
\hline \multirow{2}{*}{ W. } & \multirow[t]{2}{*}{ t. } & \multirow[t]{2}{*}{ sandy loam. } & \multirow{2}{*}{$\begin{array}{l}0-20 \mathrm{~cm} . \\
\text { under } 20\end{array}$} & \multirow{2}{*}{$\begin{array}{l}1.52 \\
0.76\end{array}$} & \multirow{2}{*}{$\begin{array}{r}13.16 \\
6.08\end{array}$} & \multirow{2}{*}{$\begin{array}{l}11.55 \\
12.15\end{array}$} \\
\hline & & & & & & \\
\hline & \multirow[t]{2}{*}{2.} & \multirow[t]{2}{*}{ sandy loam. } & $0-20$ & 0.40 & 26.29 & 1.52 \\
\hline & & & under 20 & 0.05 & 14.18 & 0.35 \\
\hline & \multirow[t]{2}{*}{3.} & \multirow{2}{*}{ loam. } & $0-20$ & 0.10 & 20.72 & 0.48 \\
\hline & & & under 20 & 0.10 & 22.60 & 0.44 \\
\hline 4. & \multirow{2}{*}{4.} & \multirow[t]{2}{*}{ loam. } & $0-20$ & 0.32 & 27.31 & 1.15 \\
\hline$"$ & & & under 20 & 0.13 & 27.15 & 0.48 \\
\hline & \multirow[t]{2}{*}{5.} & \multirow[t]{2}{*}{ clayey loam. } & $0-20$ & 0.41 & 16.29 & 2.52 \\
\hline & & & under 20 & 0.21 & 10.30 & 2.04 \\
\hline \multirow[t]{2}{*}{$\mathrm{X}$} & \multirow[t]{2}{*}{1.} & \multirow[t]{2}{*}{ loam. } & $0-20$ & 0.51 & 20.34 & 2.51 \\
\hline & & & under 20 & 0.20 & 30.55 & 0.66 \\
\hline 2. & \multirow[t]{2}{*}{2.} & \multirow[t]{2}{*}{ sandy loam. } & $0-20$ & 0.21 & 18.36 & 1.09 \\
\hline & & & under 20 & 0.20 & 28.07 & 0.73 \\
\hline 3. & . & \multirow[t]{2}{*}{ sandy loan. } & $0-20$ & 0.41 & 31.09 & 1.32 \\
\hline & & & under 20 & 0.20 & 39.92 & 0.50 \\
\hline if & & \multirow[t]{2}{*}{ loam. } & $0-20$ & 0.33 & 30.18 & 1.08 \\
\hline & & & under 20 & 0.20 & 36.49 & 0.66 \\
\hline 5. & & \multirow[t]{2}{*}{ sandy loam. } & $0-20$ & 0.20 & 5.37 & 3.72 \\
\hline & & & under 20 & 0.10 & 10.28 & 0.97 \\
\hline 6. & . & \multirow[t]{2}{*}{ sandy loam } & $0-20$ & 0.47 & 14.38 & 3.27 \\
\hline & & & under 20 & 0.13 & 6.15 & 2.11 \\
\hline 7 & & \multirow[t]{2}{*}{ sandy loatn. } & $0-20$ & 0.20 & 30.69 & 0.65 \\
\hline & & & under 20 & 0.20 & 24.40 & 0.82 \\
\hline & \multirow[t]{2}{*}{8.} & \multirow[t]{2}{*}{ sandy loam. } & $0-20$ & 0.20 & 20.39 & 0.98 \\
\hline & & & under 20 & 0.20 & 22.34 & 0.90 \\
\hline 9 & & loam. & $0-20$ & 0.33 & 5.79 & 5.70 \\
\hline & & & under 20 & 0.10 & 8.14 & 1.23 \\
\hline
\end{tabular}




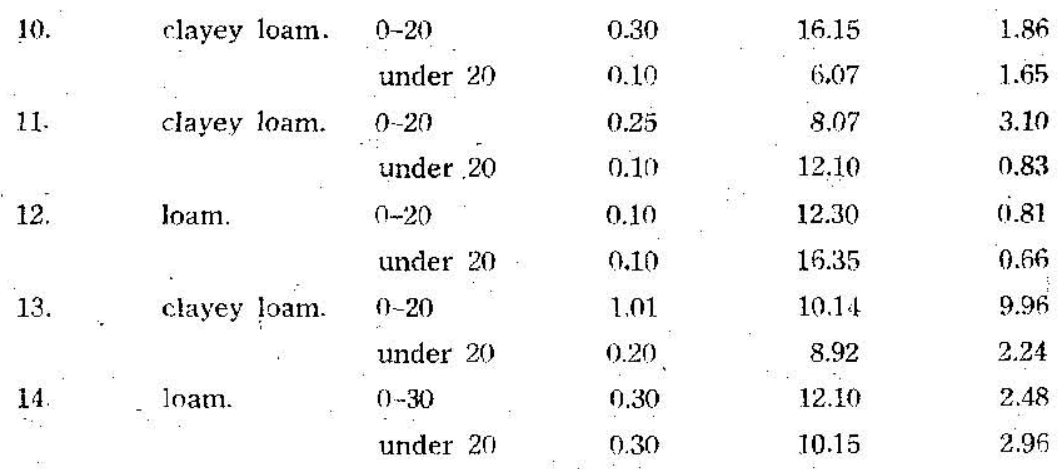

(c) Marine Soil.

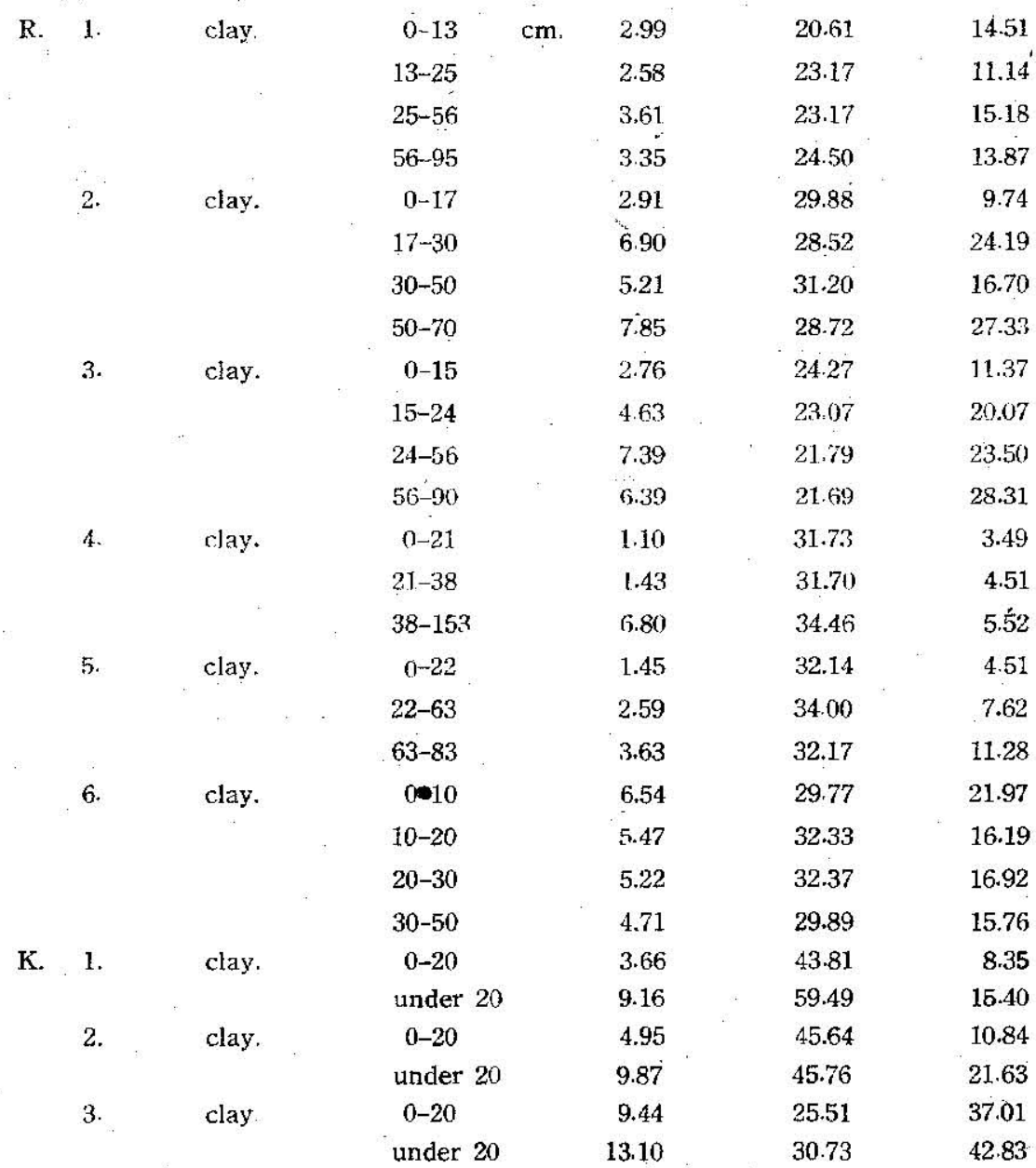


(d) Forest Soil.

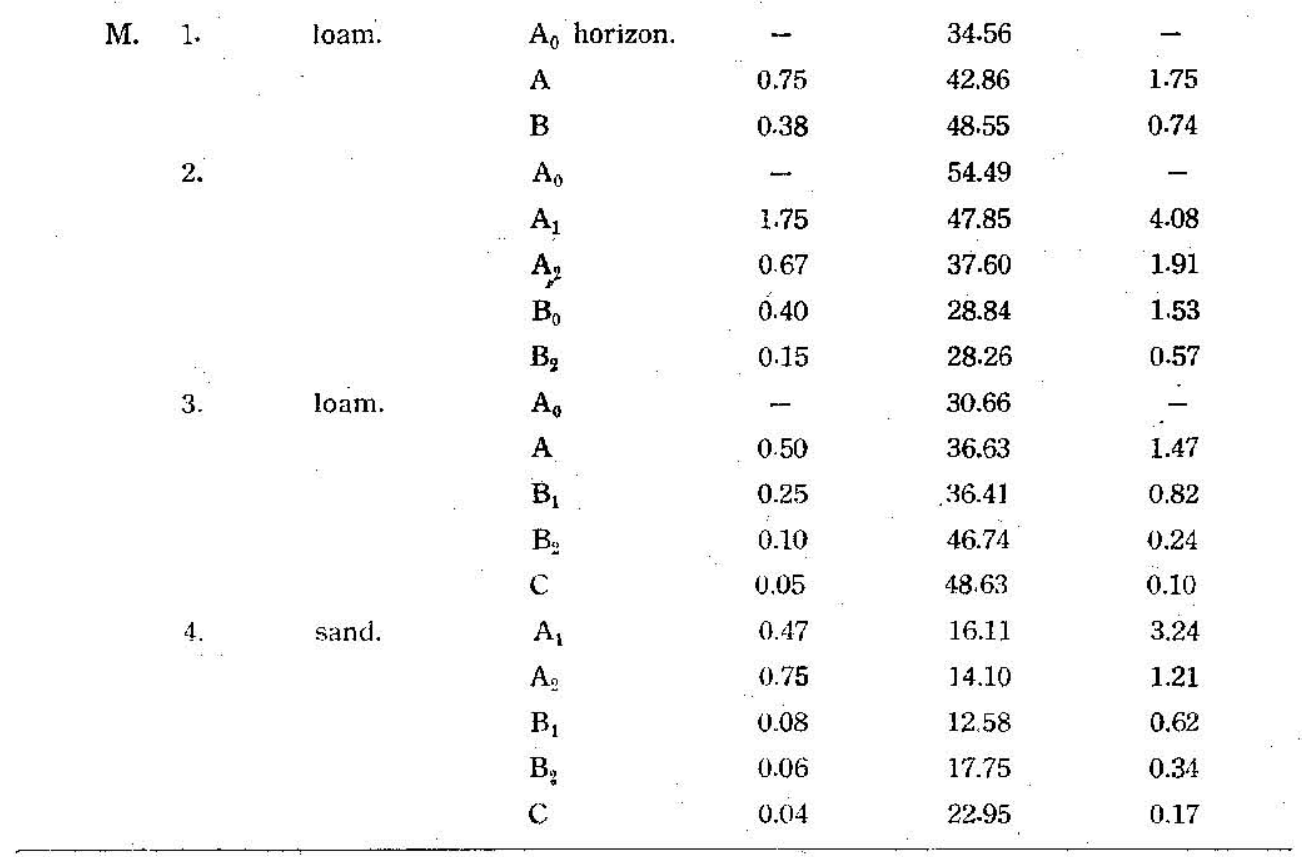

* A : available boron. $\mathrm{T}$ : total boron.

Table 2 indicates the boron content found in various representative fertilizer materials.

Table 2. Boron Content in Fertilizer Materials. (p.p.m. in dry basis.)

\begin{tabular}{lccc}
\multicolumn{2}{c}{$\begin{array}{c}\text { Bample. } \\
\text { Boron } \\
\text { Content. }\end{array}$} & Sample. & Boron \\
Sulfate of potash. & 26.4 & Straw ash. & (a) 15.16 \\
Muriate of potash. (Trona) & 466.4 & & (b) 20.83 \\
Muriate of potash. (Palestein)) & 30.8 & & (c) 21.18 \\
& & Grass ash. & (d) 16.40 \\
Nitrate of soda. (Old style) & 446.6 & & (e) 21.13 \\
& & & (b) 102.46 \\
Nitrate of soda. (Champion) & 83.6 & & (c) 160.66
\end{tabular}




\begin{tabular}{|c|c|c|c|c|c|c|}
\hline Rock phosphate. & (a) & 26.4 & & & (e) & 52.07 \\
\hline & (b) & 22.0 & & Wood ash. & (a) & 261.13 \\
\hline & (c) & 26.0 & & & (b) & 179.32 \\
\hline$\therefore$ & (d) & 20.0 & & & (c) & 169.22 \\
\hline Superphosphate. & (a) & 22.0 & & & (d) & 176.72 \\
\hline & (b) & 22.0 & & & (e) & $126 . \overline{79}$ \\
\hline Sulfate of ammonia. & & 0.0 & & Camphor-tree ash. & (a) & 84.88 \\
\hline Cyanamid. & & 00 & & & (b) & $352.28^{\circ}$ \\
\hline Guano (Peru) & & $00^{\circ}$ & & & (c) & 172.18 \\
\hline Rape seed cake. & & 13.0 & & & (d) & 130.38 \\
\hline Soybean cake. & & 180 & & & (e) & 132.23 \\
\hline Kapok seed cake. & & 13.0 & & Rice-hull ash. & (a) & 16.94 \\
\hline Cotton seed cake. & & $26.1)$ & & & (b) & 25.74 \\
\hline Pressed fish. (sardine) & & 00 & & & (c) & 20.30 \\
\hline Compost & (a) & 7.65 & & & (d) & 4.20 \\
\hline & (b) & 3.44 & & & (e) & 15.23 \\
\hline & (c) & 4.82 & & Suger cane ash. & & 40.73 \\
\hline & (d) & 3.14 & & Corn stalk ash. & & 40.75 \\
\hline & (e) & 13.22 & & Fern ash. & & 102.23 \\
\hline & (f) & 6.05 & & Tobacco ash. & (a) & 30.57 \\
\hline & (g) & 4.25 & & & (b) & 101.00 \\
\hline & (h) & 4.40 & & & (c) & 106.77 \\
\hline & (i) & 5.48 & & & (d) & 114.52 \\
\hline & & & & & (e) & 51.67 \\
\hline
\end{tabular}

Sweet poteto runner ash. $\quad 203.96$

Table 3 indicates the boron content found in various plants, vegetables, and miscellaneous samples of plant orgigin.

Table 3. Boron Content in various Plants, Vegetables, and Miscellaneous samples. (p.p.m. in dry basis.)

Sample.

(a) Leaves of trees.

Cryptomeria japonica.

(a) 20.87

(b) ${ }^{*} \quad 14.88$

Pinus Thumbergii.
Boron

Content.

(a)* 29.10
Sample.

(e) Cereals.

Rice (glutinous) unhulled rice. $\quad 0.64$
Boron

Content.

1.55 
Picea jezoensis.

Abies Mayriana.

Fagus japonica.

Cycas revolta.

(b) Marine weeds.

Entesonorpha Luiza.

88.62

Ulva pertusa.

Porphyra tenera.

Gloiopeltis furcata.

Narnaluon puloinatum.

Gracilaria confervoides.

Hisikia fusifarme.

Nematocystus decipies.

(c) Fresh water weeds

Nostoc verrucosum.

12.84

Phylloderma sacrum.

Prasiola japonica.

trace.

2.25

(a) Vegetables.

Radish. tops.

36.70

roots. $\quad 20.90$

Turnip. tops. $\quad 44.04$

roots. $\quad 29.80$

Japanese radish. tops.

31.75

14.69

Carrot. tops.

roots.

Lotus roots. roots.

Welsh onion.

33.80

16.88

10.49

15.70

$\begin{array}{ll}\text { Spinach. } & 37.97 \\ \text { Chinese cabtıge. } & 18.48\end{array}$

$\begin{array}{ll}\text { Spinach. } & 37.97 \\ \text { Chinese cablıge. } & 18.48\end{array}$ straw.

1.41

Rice (non-glutinous)

unhulled rice. $\quad 0.50$

rice-hulls. $\quad 1.55$

straw. $\quad 0.88$

Barley, grain. $\quad 1.87$

straw. $\quad 1.77$

Wheat. grain. 2.11

straw. 3.41

(f) Micellaneous amples.

Tobacco. (burley)

leaves. $\quad 48.12$

stalks. $\quad 11.74$

flowers. $\quad 2825$

Apples. (1) leaves. $\quad 26.10$

fruit. $\quad 9.99$

(2) leaves. $\quad 51.66$

fruit. $\quad \mathbf{1 1 8 3}$

(3) leaves. $\quad 18.15$

fruit. $\quad 3.78$

(4) leaves. $\quad 56.34$

fruit. $\quad 25.30$

(5) leaves. $\quad 38.10$

fruit. $\quad 17.40$

$(a)^{*}$ : fresh leaves. (b) ${ }^{*}$ fallen legaves. 


\section{Summary and Conclusion.}

Robinson and his co-workers (2) reported that in forty-two samples of American soils, the total boron content ranged from 64 to 65.2 p.p.m. in dry basis and soluble boron (in phosphoric acid from 1.0 to $45.5 \mathrm{p} . \mathrm{p} . \mathrm{m}$. in ninety one samples.

Suijakova (3) found that in fifteen samples of U.S.S.R. soils, the total boron content ranged from 2.0 in 160 p.p.m. and water soluble boron from 0.19 to 1.10 p.p.m.

Amounts detectrible in Japanese soils as observed by the author are in table 1 . Viewing these results, it is evident that soils vary widely in their content of boron.

A brief summary of these studies is as follows.

$\begin{array}{lccc} & \begin{array}{c}\text { Samples } \\ \text { examined. }\end{array} & \begin{array}{c}\text { Total } \\ \text { Boron }\end{array} & \begin{array}{c}\text { Available } \\ \text { Boron. }\end{array} \\ \text { Virgin soil. } & 32 & 1.46-39.3 \times & 0.05-0.38 \\ \text { Arable soil. } & 38 & 5.37-39.92 & 0.05-1.52 \\ \text { Fiuvial soil. } & 28 & 5.37-39.92 & 0.10-1.01 \\ \text { Marine soil. } & 28 & 20.61-59.49 & 1.10-13.10 \\ \text { Forest soil. } & 18 & 12.58-48.55 & 0.08-1.75\end{array}$

In virgin soil, half of a sample contains total boron $10-20$ p.p.m. in dry basis and seventy-five percent of a sample contains it in available form $0.1-0.3$ p.p.m.

But in arable soil, sixty-three percent of a sample contains total boron $10-30$ p.p.m. and available form $0.1-0.3$ p.p.m. In the upper layer $(0-10 \mathrm{~cm}$. deep) of arable soil, sixty-three percent of a sample contains available boron over 0.3 p.p.m but in the subsoil (under $10 \mathrm{~cm}$. deep) eighty-three percent of a sample contains it at $0.1-0.3$ p.p.m.

The greatest amount of boron in available form is found in marine soil; $1.10-13.10$ p.p.m.

As a rule, no definite relation exists between the total and the available amounts of boron. In all samples, except marine soil, the available boron centent is less in the subsoil strata.

As table 2 shows, fertilizer materials of natural origin genarally 
contain a high boron content.

Since the boron content of various ashes is constantly high, application of these substances as potassium fertilizer will probably increase the boron content of the soil.

Plants contain varying amounts of boron and the smallest amount is found in cereals. In vegetables, it is present in greater amounts in the tops than in the roots. All sea weeds except one type contain large amounts of boron, but in water weeds, the amount is very small.

\section{REFERENCES :}

(1) Berger, K. C. and Troug, E.: Ind. Eng. Chem. Anal. Ed. 11. p. 540. (1939)

(2) Robinson, W. O. Weston, R. R. and Byers, H. G.: Amer. Fertilizer, 91. p. 7 (1939)

(3) Suijakova, S. I.: Chem. Abstr. 34. 4202 (1940) 